\title{
Formal Total Synthesis of (-)-Emetine Using Catalytic Asymmetric Allylation of Cyclic Imines
}

\section{as a Key Step}

Takashi Itoh*, Michiko Miyazaki, Hiromi Fukuoka, Kazuhiro Nagata, and Akio Ohsawa

\section{Supporting Information}

The synthesis of 2: To the THF solution ( $3 \mathrm{ml})$ of $\mathrm{CuCl}(20 \mathrm{mg}, 10 \mathrm{~mol} \%)$ were added $(R)$-Tol-BINAP $(136 \mathrm{mg}, 10 \mathrm{~mol} \%)$ and tetrabutylammonium difluorotriphenylsilicate $(110 \mathrm{mg}, 10 \mathrm{~mol} \%)$, and the mixture was allowed to stir for $1 \mathrm{~h}$ at room temperature under Ar. After 3,4-dihydro-6,7-dimethoxyisoquinoline (1) $(382 \mathrm{mg}, 2 \mathrm{mmol})$ and allyltrimethylsilane (0.68 $\mathrm{ml}, 4 \mathrm{mmol})$ were added to the mixture at $0^{\circ} \mathrm{C}, t-\mathrm{BuOH}(200 \mu \mathrm{l})$ was added at roome temperature, and the mixture was further reacted for $1 \mathrm{~d}$. The reaction was stopped by the addition of sat. $\mathrm{NaHCO}_{3}(100 \mathrm{ml})$, then brine $(50 \mathrm{ml})$ was added, and the mixture was extracted with AcOEt. The organic layer thus obtained was dried over $\mathrm{MgSO}_{4}$, and evaporated off. The residue was chromatographed on silica gel to give the product $(S)$-2 (424 $\mathrm{mg}, 91 \%)$. The ee of $\mathbf{2}(71 \%)$ was determined by HPLC after trifluoroacetylation of N-2 position of 2.

Recrystallization of 2 in the presence of (-)-dibenzoyltartaric acid: The product ( $127.3 \mathrm{mg}, 0.53 \mathrm{mmol})$ obtained by the above reaction and (-)-dibenzoyl tartrate (190 mg, $0.53 \mathrm{mmol}$ ) was mixed in the $\mathrm{CH}_{3} \mathrm{CN} / \mathrm{H}_{2} \mathrm{O}$ (20) solution (3.7 ml). The solvent was evaporated under reduced pressure, and the residue was recrystallized with $\mathrm{CH}_{3} \mathrm{CN} / \mathrm{H}_{2} \mathrm{O}(20)$. The precipitate was filtered, and the filtrate was suspended in $10 \mathrm{ml}$ of $\mathrm{H}_{2} \mathrm{O}$. The aqueous solution was alkalized with $\mathrm{Na}_{2} \mathrm{CO}_{3}$, and extracted with AcOEt. The organic layer was dried over $\mathrm{MgSO}_{4}$, and evaporated to leave an optically pure $(S)-2(99.3 \mathrm{mg}$, 78\%). The optical purity was determined by HPLC analysis (CHIRALCELOD, hexane/i-PrOH=2, $0.5 \mathrm{ml} / \mathrm{min}$, retention times $14.9 \mathrm{~min}$ and 17.7 min) as $97 \%$ ee. 


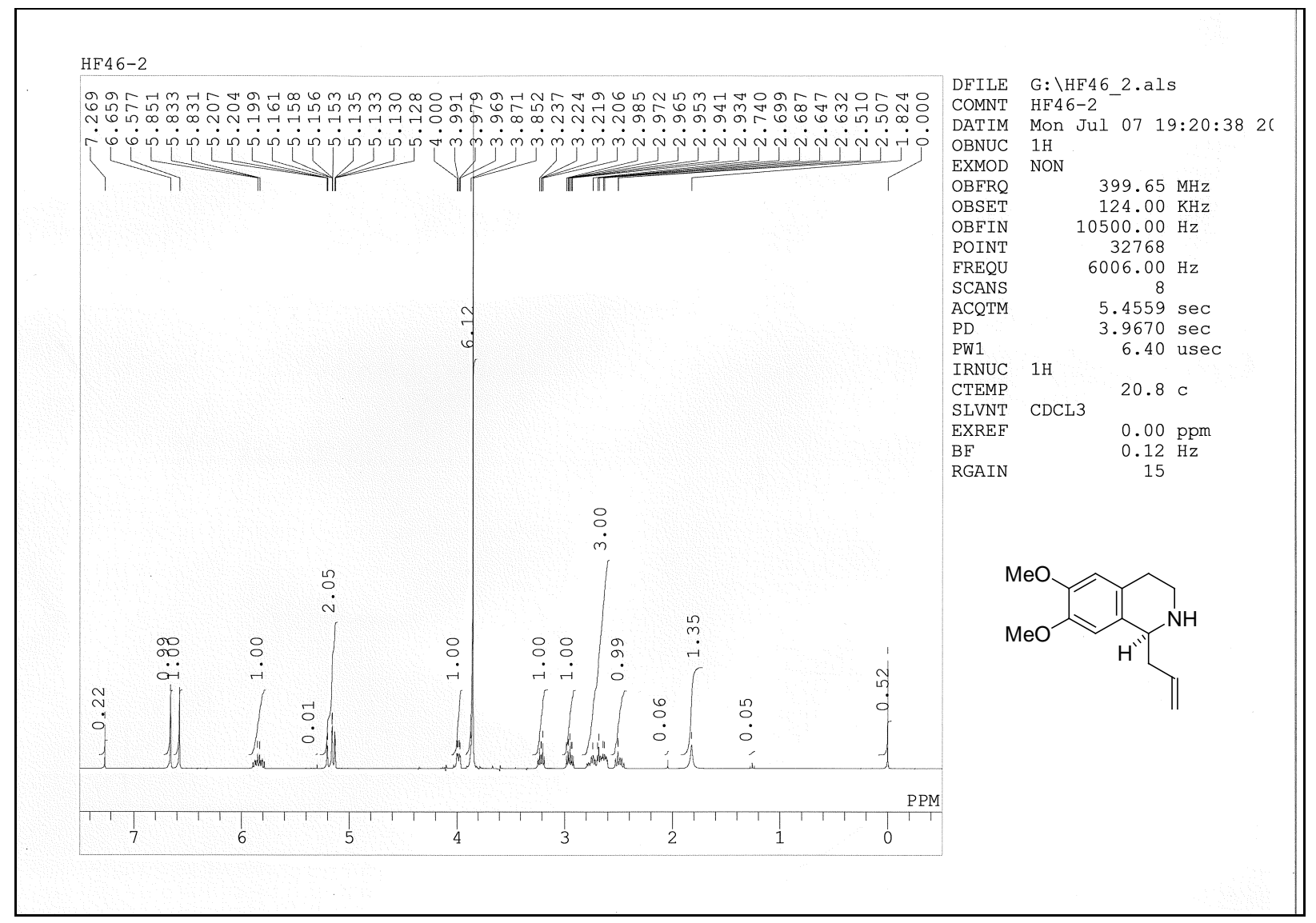

\section{1-allyl-6,7-dimethoxy-1,2,3,4-tetrahydroisoquinoline}

Yield $91 \%$; Yellow needles mp $33-36{ }^{\circ} \mathrm{C}$

$R$-(98.7\% ee) : $[\alpha]^{18} \mathrm{D}-81.4\left(c 0.44, \mathrm{CHCl}_{3}\right)$

$S-(99.8 \%$ ee $):[\alpha]^{17}{ }_{D}+83.4\left(c 1.66, \mathrm{CHCl}_{3}\right)$

${ }^{1} \mathrm{H}-\mathrm{NMR}\left(\mathrm{CDCl}_{3}\right) \delta: 1.82(1 \mathrm{H}, \mathrm{bs}), 2.49(1 \mathrm{H}, \mathrm{dtd}, J=15.3,8.0,1.0 \mathrm{~Hz}), 2.60-2.67(1 \mathrm{H}, \mathrm{m}), 2.67-2.79(2 \mathrm{H}, \mathrm{m}), 2.95(1 \mathrm{H}, \mathrm{ddd}, J=$ 12.6, 7.8, $4.9 \mathrm{~Hz}), 3.22(1 \mathrm{H}, \mathrm{dt}, J=12.3,5.3 \mathrm{~Hz}), 3.85(6 \mathrm{H}, \mathrm{s}), 4.00(1 \mathrm{H}, \mathrm{dd}, J=8.7,3.6 \mathrm{~Hz}), 5.13-5.21(2 \mathrm{H}, \mathrm{m}), 5.79-5.89(1 \mathrm{H}, \mathrm{m})$, $6.58(1 \mathrm{H}, \mathrm{s}), 6.66(1 \mathrm{H}, \mathrm{s})$.

${ }^{13} \mathrm{C}-\mathrm{NMR}\left(\mathrm{CDCl}_{3}\right) \delta ; 29.5,40.8,41.1,54.7,55.8,56.0,56.0,109.1,111.8,117.9,127.5,130.5,135.6,147.2,147.4$.

Anal. Calcd for $\mathrm{C}_{14} \mathrm{H}_{19} \mathrm{NO}_{2}$ : C,72.07; H, 8.21; N, 6.00. Found: C, 71.81, H, 8.34; N, 5.94.

HR-FAB MS : Calcd for $\mathrm{C}_{14} \mathrm{H}_{20} \mathrm{NO}_{2}[\mathrm{M}+\mathrm{H}]^{+}: 234.1494$. Found 234.1480. 
The synthesis of 1-allyl-6,7-dimethoxy-3,4-dihydro-1H-isoquinoline-2-carboxylic acid tert-butyl ester 3 To the $\mathrm{CH}_{2} \mathrm{Cl}_{2}$ solution $(8 \mathrm{ml})$ of $(\mathrm{Boc})_{2} \mathrm{O}(580.2 \mathrm{mg}, 2.5 \mathrm{mmol})$ was added the $\mathrm{CH}_{2} \mathrm{Cl}_{2}$ solution $(7 \mathrm{ml})$ of copmpound $2(307.7 \mathrm{mg}, 1.3 \mathrm{mmol})$, and the mixture was allowed to react for $1 \mathrm{~h}$ at room temperature under $\mathrm{Ar}$. The raction was stopped by the addition of $\mathrm{H}_{2} \mathrm{O}(30$ $\mathrm{ml}$ ), and the mixture was extracted with $\mathrm{CH}_{2} \mathrm{Cl}_{2}$. The organic layer thus obtained was dried over $\mathrm{MgSO}_{4}$, and evaporated to leave a residue, which was chromatographed on silica gel $\left(\mathrm{CH}_{2} \mathrm{Cl}_{2} / \mathrm{AcOEt}=20\right)$ to give 3 (432.5 mg, 98\%).

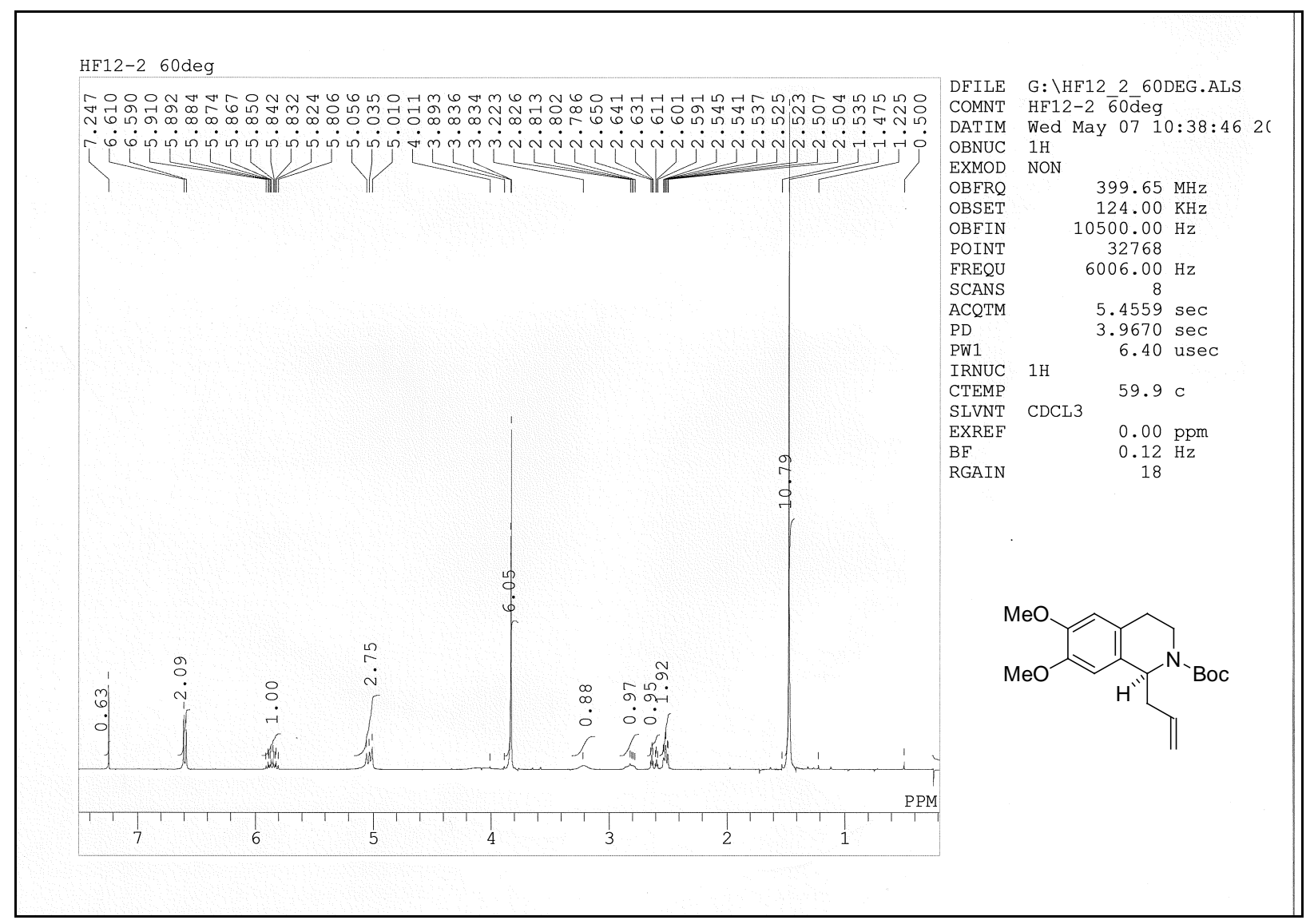

1-allyl-6,7-dimethoxy-3,4-dihydro-1H-isoquinoline-2-carboxylic acid tert-butyl ester

Yield $97 \%$ colorless powder

$R-(98.7 \%$ ee $):[\alpha]^{18}-81.4\left(c 0.44, \mathrm{CHCl}_{3}\right)$

$S-(99.8 \%$ ee $):[\alpha]^{17}+83.4\left(c 1.66, \mathrm{CHCl}_{3}\right)$

${ }^{1} \mathrm{H}-\mathrm{NMR}\left(\mathrm{DMSO}-\mathrm{d}_{6}, 100^{\circ} \mathrm{C}\right) \delta: 1.44(9 \mathrm{H}, \mathrm{s}), 2.45-2.57(2 \mathrm{H}, \mathrm{m}), 2.65(1 \mathrm{H}, \mathrm{dt}, J=16.2,4.2 \mathrm{~Hz}), 2.72(1 \mathrm{H}, \mathrm{ddd}, J=16.2,10.4,5.8$

Hz), 3.19 (1H, ddd, J=13.1, 10.4, $4.3 \mathrm{~Hz}), 3.74(3 \mathrm{H}, \mathrm{s}), 3.75(3 \mathrm{H}, \mathrm{s}), 3.94(1 \mathrm{H}, \mathrm{dt}, J=12.8,4.5 \mathrm{~Hz}), 4.99-5.06(3 \mathrm{H}, \mathrm{m}), 5.82$ (1H, ddt, $J=17.1,10.1,7.1 \mathrm{~Hz}), 6.70(1 \mathrm{H}, \mathrm{s}), 6.77(1 \mathrm{H}, \mathrm{s})$. 
${ }^{13} \mathrm{C}-\mathrm{NMR}\left(\mathrm{DMSO}-\mathrm{d}_{6}, 100^{\circ} \mathrm{C}\right) \delta$; 27.2, 27.8, 37.2, 40.4, 53.2, 55.7, 55.9, 78.6, 111.5, 112.8, 126.1, 129.1, 135.1, 147.4, 147.7, 153.8 .

Anal. Calcd for $\mathrm{C}_{19} \mathrm{H}_{27} \mathrm{NO}_{4}$ : C,68.44; H, 8.16; N, 4.20. Found: C, 68.43, H, 8.37; N, 4.04.

HR-FAB MS : Calcd for $\mathrm{C}_{19} \mathrm{H}_{28} \mathrm{NO}_{4}[\mathrm{M}+\mathrm{H}]^{+}$: 334.2018. Found 334.2029.

The synthesis of 6,7-dimethoxy-1-(3-ethoxycarbonylallyl)-3,4-dihydro-1H-isoquinoline-2-carboxylic acid tert-butyl ester 4.

To the $\mathrm{CH}_{2} \mathrm{Cl}_{2}$ solution $(3 \mathrm{ml})$ of compound $3(67 \mathrm{mg}, 0.2 \mathrm{mmol})$ were added ethyl acrylate $(150 \mu \mathrm{l}, 1 \mathrm{mmol})$ and the Grubbs catalyst (5.3 mg, $0.006 \mathrm{mmol})$, and the mixture was allowed to react for $4 \mathrm{~h}$ at $50^{\circ} \mathrm{C}$ under argon. $\mathrm{Then} \mathrm{CH}_{2} \mathrm{Cl}_{2}(5 \mathrm{ml})$ and $\mathrm{Al}_{2} \mathrm{O}_{3}$ (100 mg) were added, and the mixture was allowed to stir for $30 \mathrm{~min}$ at room temperature. The mixture was filtrated, and the solid thus separated was washed with AcOEt. The organic layers were combined, and evaporated off. The residue was chromatographed on silica gel to give the product (75 mg, 93\%)

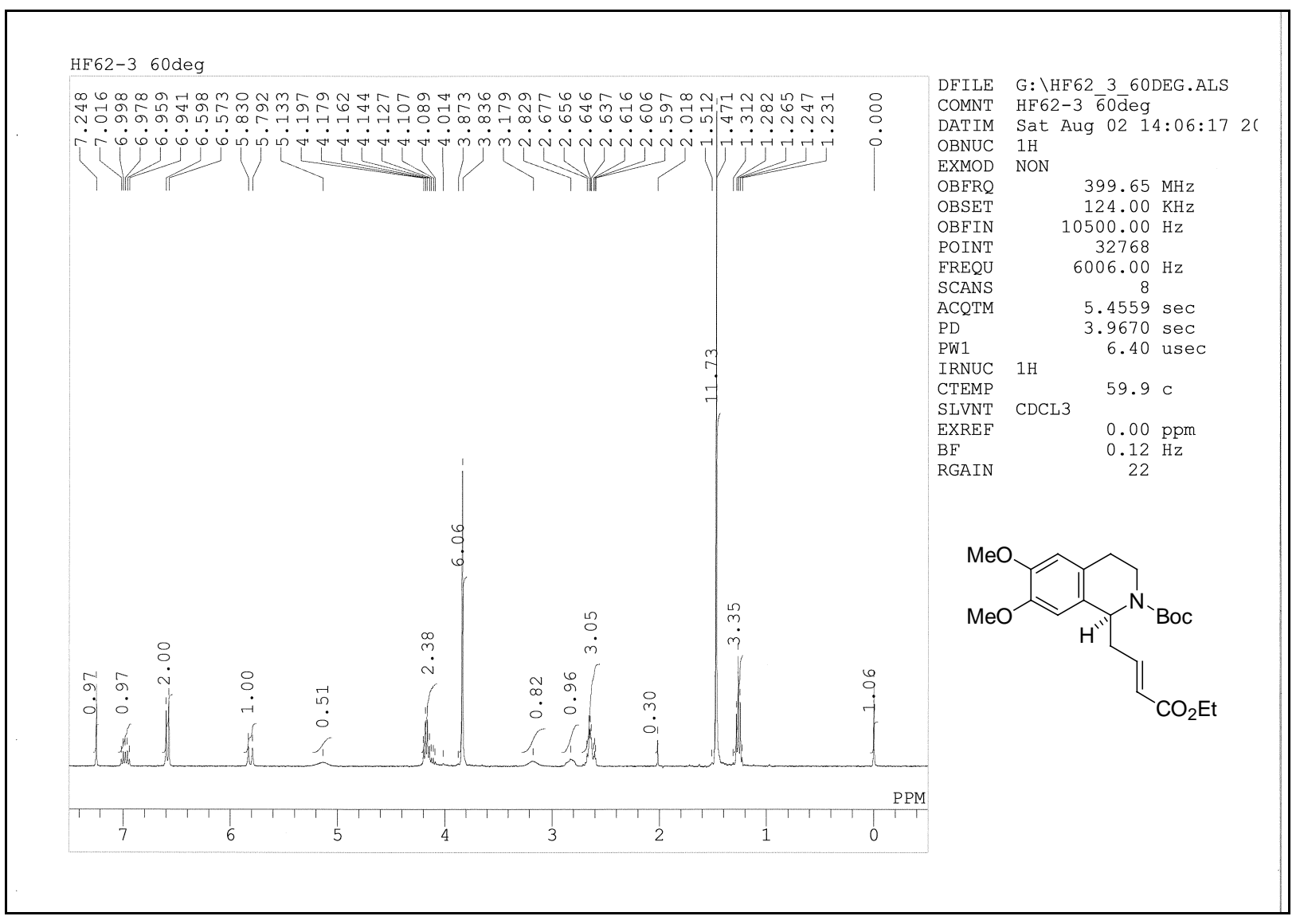

S 4 


\section{6,7-dimethoxy-1-[3-(ethoxycarbonyl)allyl]-3,4-dihyhdro-1H-isoquinoline-2-carbolic acid tert-butyl ester}

Yield $97 \%$ yellow oil

$R-(98.7 \%$ ee $):[\alpha]^{18}-81.4\left(c 0.44, \mathrm{CHCl}_{3}\right)$

$S-(99.8 \%$ ee $):[\alpha]^{17}{ }^{17}+83.4\left(c 1.66, \mathrm{CHCl}_{3}\right)$

${ }^{1} \mathrm{H}-\mathrm{NMR}\left(\mathrm{CDCl}_{3}, 60^{\circ} \mathrm{C}\right) \delta: 1.26(3 \mathrm{H}, \mathrm{t}, J=7.1 \mathrm{~Hz}), 1.47(9 \mathrm{H}, \mathrm{s}), 2.60-2.72(3 \mathrm{H}, \mathrm{m}), 2,83(1 \mathrm{H}, \mathrm{bs}), 3.18(1 \mathrm{H}, \mathrm{bs}), 3.84(6 \mathrm{H}, \mathrm{s}), 4.17$

$(2 \mathrm{H}, \mathrm{q}, J=7.1 \mathrm{~Hz}), 4.20(1 \mathrm{H}, \mathrm{bs}), 5.13(1 \mathrm{H}, \mathrm{bs}), 5.81(1 \mathrm{H}, \mathrm{d}, J=15.4 \mathrm{~Hz}), 6.57(1 \mathrm{H}, \mathrm{s}), 6.60(1 \mathrm{H}, \mathrm{s}), 6.98(1 \mathrm{H}, \mathrm{dt}, J=15.3,7.6 \mathrm{~Hz})$.

${ }^{13} \mathrm{C}-\mathrm{NMR}\left(\mathrm{CDCl}_{3}, 6{ }^{\circ} \mathrm{C}\right) \delta ; 14.2,28.2,28.4,36.0,39.8,53,9,56.1,60.1,80.0,110.6,112.2,123.6,126.8,128.6,145.0,147.9$, $148.3,154.5,166.0$.

Anal. Calcd for $\mathrm{C}_{22} \mathrm{H}_{31} \mathrm{NO}_{6}$ : C,65.17; H, 7.71; N, 3.45. Found: C, 65.37, H, 7.78; N, 3.22.

HR-FAB MS : Calcd for $\mathrm{C}_{22} \mathrm{H}_{32} \mathrm{NO}_{6}[\mathrm{M}+\mathrm{H}]^{+}$: 406.2223. Found 406.2230.

The synthesis of 4-(6,7-diemethoxy-1,2,3,4-tetrahydroisoquinolin-1-yl)but-2-enoic acid ethyl ester 5 . To the $\mathrm{CH}_{2} \mathrm{Cl}_{2}$ solution $(30 \mathrm{ml})$ of compound $4(386.7 \mathrm{mg}, 0.95 \mathrm{mmol})$ was added trimethylsilyl triflate $(760 \mu \mathrm{l}, 4.2 \mathrm{mmol})$ dropwise, and the mixture was allowed to stir at room temperature in air. Then $10 \mathrm{ml}$ of sat. $\mathrm{NaHCO}_{3}$ was added, and the mixture was extracted with $\mathrm{CH}_{2} \mathrm{Cl}_{2}$ and AcOEt. The organic layer was dried over $\mathrm{MgSO}_{4}$, and evaporated to leave a residue, which was chromatographed on NH silica gel (AcOEt) to give 5 (273.4 mg, 96\%). The optical purity was determined by HPLC (CHIRALCEL OD, hexane/i-PrOH=1/2, $0.5 \mathrm{ml} / \mathrm{min}$, retention times $16.6 \mathrm{~min}$ and $22.5 \mathrm{~min}$ ). 


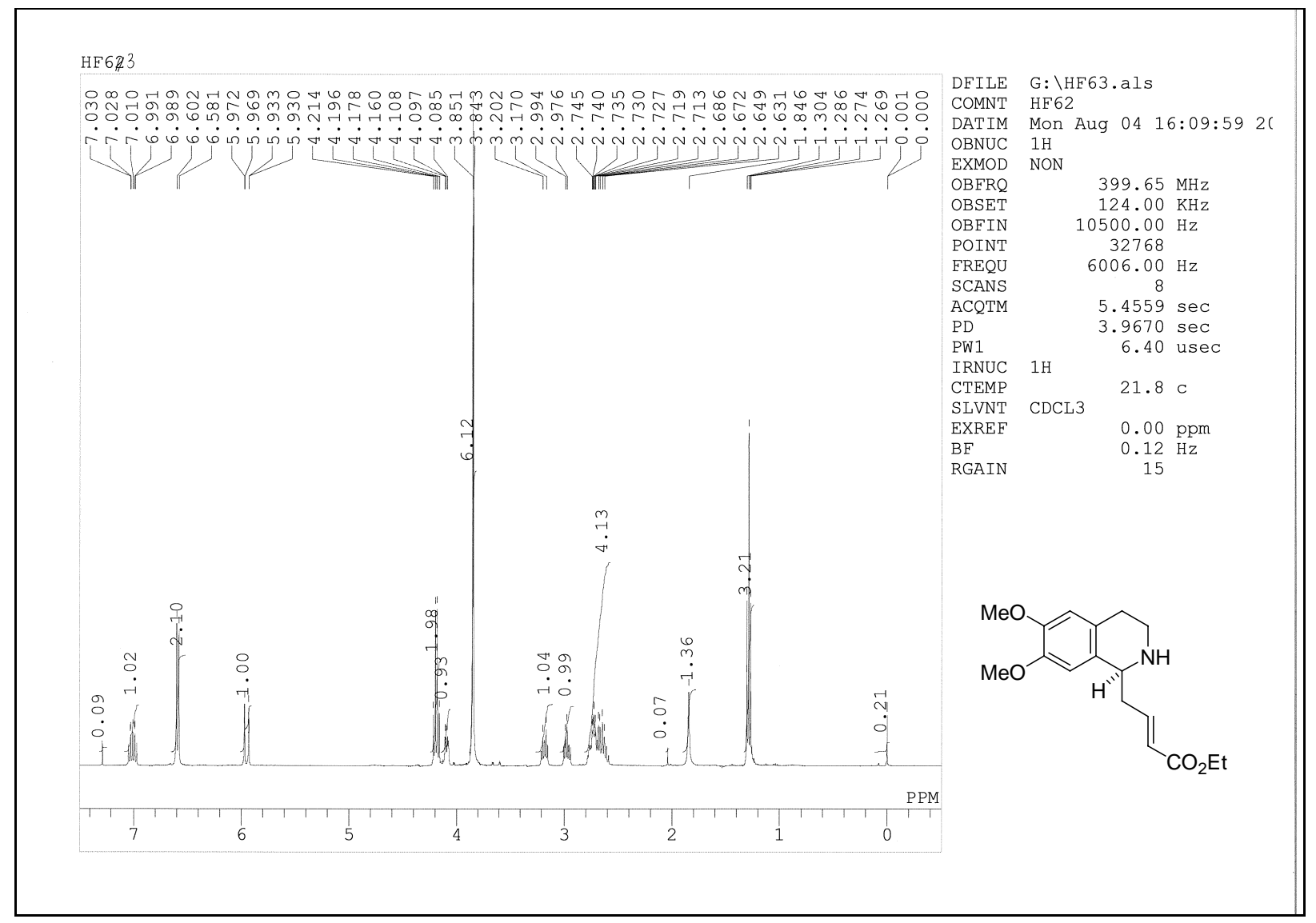

4-(6,7-dimethoxy-1,2, 3,4-tetrahydroisoquinolin-1-yl)-but-2-enoic acid ethyl ester

Yield $96 \%$ yellow crystal : $\mathrm{mp} 61-63^{\circ} \mathrm{C}$

$R-(98.5 \%$ ee $):[\alpha]^{18}+85.5\left(c 0.76, \mathrm{CHCl}_{3}\right)$

$S-(99.7 \%$ ee $):[\alpha]^{17}$ D $-87.2\left(c 1.66, \mathrm{CHCl}_{3}\right)$

${ }^{1} \mathrm{H}-\mathrm{NMR}\left(\mathrm{CDCl}_{3}\right) \delta: 1.29(3 \mathrm{H}, \mathrm{t}, J=7.1 \mathrm{~Hz}), 1.85(1 \mathrm{H}, \mathrm{bs}), 2.59-2.78(4 \mathrm{H}, \mathrm{m}), 2,98(1 \mathrm{H}, \mathrm{ddd}, J=12.5,7.1,5.1 \mathrm{~Hz}), 3.19(1 \mathrm{H}, \mathrm{dt}$, $J=12.1,5.9 \mathrm{~Hz}), 3.84(3 \mathrm{H}, \mathrm{s}), 3.85(3 \mathrm{H}, \mathrm{s}), 4.09(1 \mathrm{H}, \mathrm{dd}, J=8.9,4.1 \mathrm{~Hz}), 4.19(2 \mathrm{H}, \mathrm{q}, J=7.1 \mathrm{~Hz}), 5.95(1 \mathrm{H}, \mathrm{d}, J=15.8 \mathrm{~Hz}), 6.58$ (1H, s), $6.60(1 \mathrm{H}, \mathrm{s}), 7.01(1 \mathrm{H}, \mathrm{ddd}, J=15.4,8.1,7.1 \mathrm{~Hz})$.

${ }^{13} \mathrm{C}-\mathrm{NMR}\left(\mathrm{CDCl}_{3}\right) \delta ; 14.2,29.2,39.3,40.7,54.5,55.8,60.3,109.0,111.9,124.0,127.4,129.6,145.9,147.2,147.6,166.2$. Anal. Calcd for $\mathrm{C}_{17} \mathrm{H}_{23} \mathrm{NO}_{4}$ 1/2 $\mathrm{H}_{2} \mathrm{O}$ : C,64.95; H, 7.69; N, 4.46. Found: C, 65.16, H, 7.63; N, 4.33.

HR-FAB MS : Calcd for $\mathrm{C}_{17} \mathrm{H}_{24} \mathrm{NO}_{4}[\mathrm{M}+\mathrm{H}]^{+}:$306.1694. Found 306.1705. 
The synthesis of (3-formyl-9,10-dimethoxy-1,3,4,6,7,11b-hexahydro-2H-pyrido[2,1-a]isoquinolin-2-yl)acetic acid ethyl ester 8. To the $\mathrm{CH}_{2} \mathrm{Cl}_{2}$ solution (15 ml) of compound 5 (305 $\left.6 \mathrm{mg}, 1.0 \mathrm{mmol}\right)$ was added pyrrolidine $(100 \mu \mathrm{l}, 1.2 \mathrm{mmol})$, and the $\mathrm{CH}_{2} \mathrm{Cl}_{2}$ solution $(15 \mathrm{ml})$ of acrolein $(380 \mu \mathrm{l}, 5.7 \mathrm{mmol})$ was added to the solution over $5.0 \mathrm{~h}$ at room temperature under Ar. The solvent was evaporated off to leave a residue, which was chromatographed on silica gel (AcOEt to AcOEt/MeOH=10) to give a cyclic product $\mathbf{8}(302.7 \mathrm{mg}, 84 \%)$.

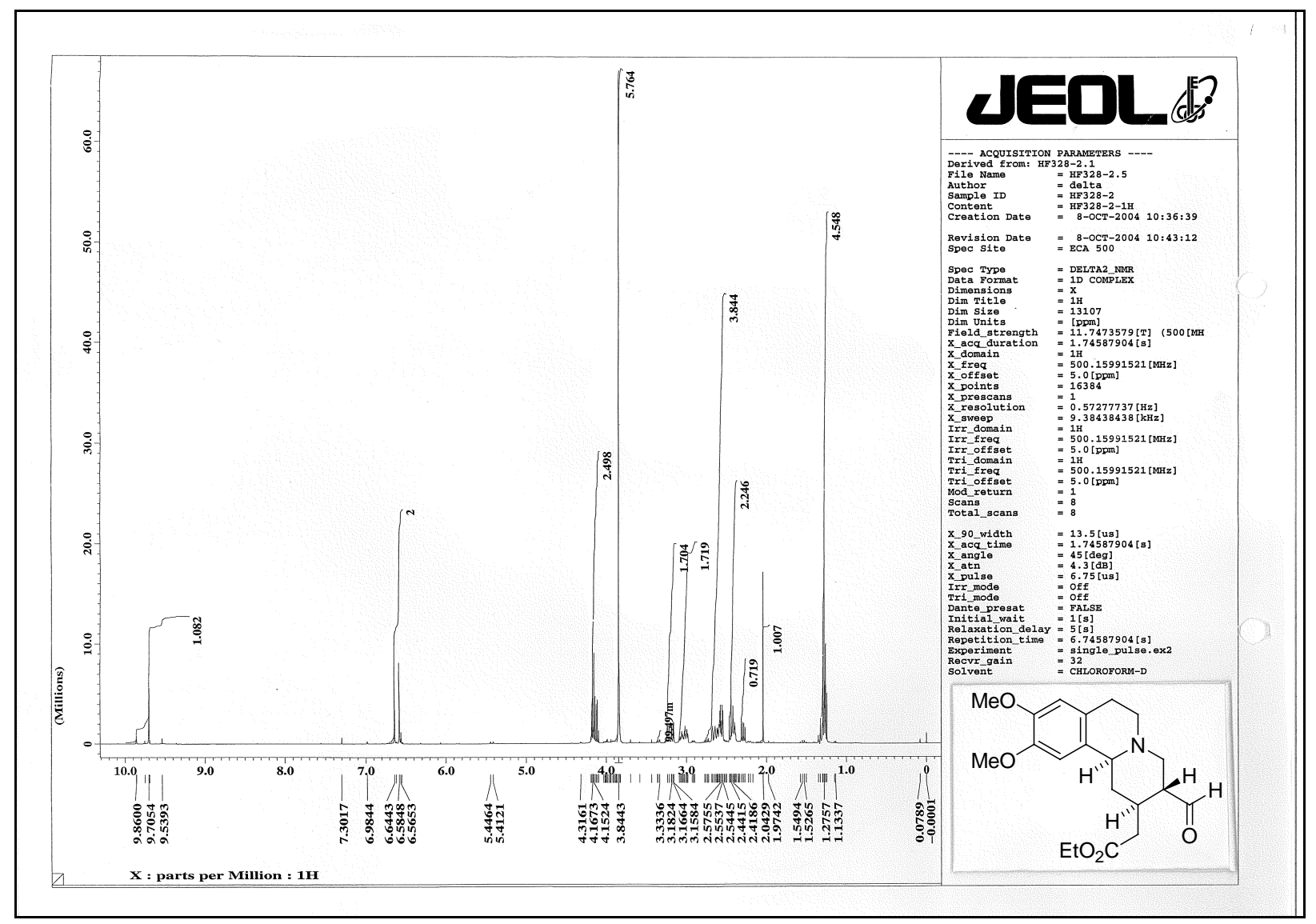

(3-formyl-9,10-dimethoxy-1,3,4,6,7,11b-hexahydro-2H-pyrido[2,1-a]isoquinolin-2-yl)acetic acid ethyl ester

Yield $84 \%$ yellow oil

$R-(98.5 \%$ ee $):[\alpha]^{18}+85.5\left(c 0.76, \mathrm{CHCl}_{3}\right)$

$S-(99.7 \%$ ee $):[\alpha]^{17}$ D $-87.2\left(c 1.66, \mathrm{CHCl}_{3}\right)$

${ }^{1} \mathrm{H}-\mathrm{NMR}\left(\mathrm{CDCl}_{3}\right) \delta: 1.25-1.35(4 \mathrm{H}, \mathrm{m}), 2.29(1 \mathrm{H}, \mathrm{dd}, J=15.8,7.7 \mathrm{~Hz}), 2.38-2.46(3 \mathrm{H}, \mathrm{m}), 2.54-2.69$ (4H, m), $2.54-2.69(4 \mathrm{H}, \mathrm{m})$, 2.98-3.03 (1H, m), 3.07 (1H, td, $J=10.9,5.7 \mathrm{~Hz}), 3.17(1 \mathrm{H}, \mathrm{dd}, J=12.0,4.0 \mathrm{~Hz}), 3.23(1 \mathrm{H}, \mathrm{d}, J=10.9 \mathrm{~Hz}), 3.84(6 \mathrm{H}, \mathrm{s}), 4.16(2 \mathrm{H}$, q, $J=7.1 \mathrm{~Hz}), 6.58(1 \mathrm{H}, \mathrm{s}), 6.64(1 \mathrm{H}, \mathrm{s}), 9.73(1 \mathrm{H}, \mathrm{d}, J=2.9 \mathrm{~Hz})$. 
${ }^{13} \mathrm{C}-\mathrm{NMR}\left(\mathrm{CDCl}_{3}\right) \delta ; 14.1,29.0,33.1,36.3,38.6,51.5,53.2,55.0,55.7,55.9 .60 .4,61.5,108.2,111.4,126.3,128.8,147.2,147.5$,

171.9, 202.4 .

Anal. Calcd for $\mathrm{C}_{20} \mathrm{H}_{27} \mathrm{NO}_{5}$ : C,66.46; H, 7.53; N, 3.88. Found: C, 66.23, H, 7.95; N, 3.53 .

HR-FAB MS : Calcd for $\mathrm{C}_{20} \mathrm{H}_{28} \mathrm{NO}_{5}[\mathrm{M}+\mathrm{H}]^{+}$: 362.1967. Found 362.1966.

The Wittig reaction of 8. To the suspension of $\mathrm{Ph}_{3} \mathrm{PCH}_{3} \mathrm{Br}(470 \mathrm{mg}, 1.32 \mathrm{mmol})$ in $\mathrm{THF}(7 \mathrm{ml}), 0.97 \mathrm{M} \mathrm{PhLi}$ solution in hexane was added at $0{ }^{\circ} \mathrm{C}$ under Ar until the reaction mixture turned yellow. Then additional PhLi slution $(1.4 \mathrm{ml})$ was introduced to the mixture at $0^{\circ} \mathrm{C}$, and the reaction was continued for $40 \mathrm{~min}$ at room temperature. After cooling to $0{ }^{\circ} \mathrm{C}$, compound $8(195.0 \mathrm{mg}$, $0.54 \mathrm{mmol})$ in THF ( $5 \mathrm{ml}$ ) was added to the mixture, and the stirring was continued for $5 \mathrm{~h}$. After MeOH $(12 \mathrm{ml})$ was added, the reaction solvent was evaporated off to leave a residue, which was chromatographed on $\mathrm{NH}$ silica gel $\left(\mathrm{CH}_{2} \mathrm{Cl}_{2}\right)$ to give the product 9 as colorless powder.

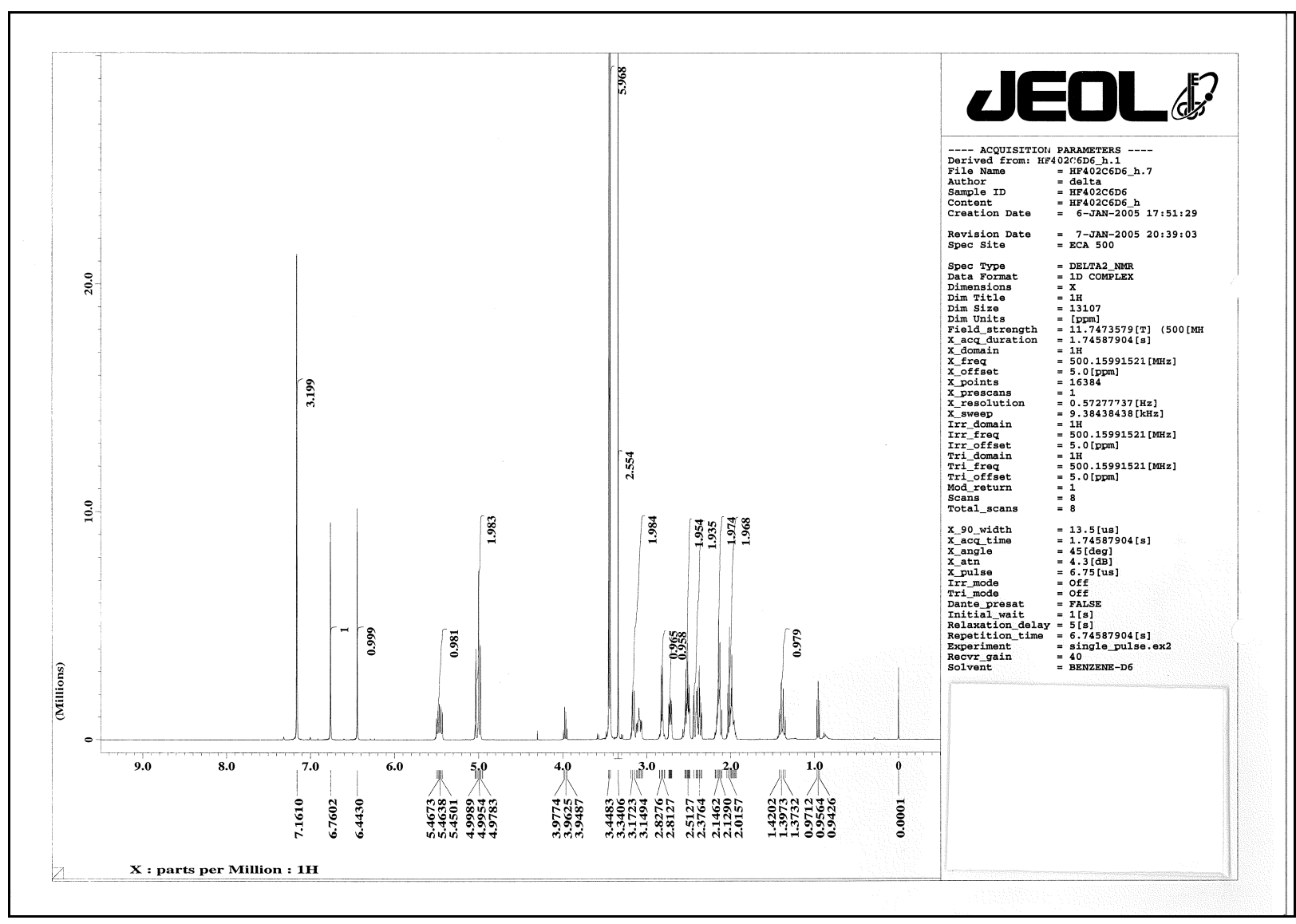


(9.10-dimethoxy-3-vinyl-1,3,4,6,7,11b-hexahydro-2H-pyrido[2,1-a]isoquinoline-2-yl)acetic acid methyl ester

Yield $57 \%$ yellow crystal : $\mathrm{mp} 98-102{ }^{\circ} \mathrm{C}$

$R-(98.5 \%$ ee $):[\alpha]^{18}{ }_{\mathrm{D}}+85.5\left(c 0.76, \mathrm{CHCl}_{3}\right)$

$S-(99.7 \%$ ee $):[\alpha]^{17}{ }^{17}-87.2\left(c 1.66, \mathrm{CHCl}_{3}\right)$

${ }^{1} \mathrm{H}-\mathrm{NMR}\left(\mathrm{C}_{6} \mathrm{D}_{6}\right) \delta: 1.39(1 \mathrm{H}, \mathrm{q}, J=11.6 \mathrm{~Hz}), 1.93-2.05(2 \mathrm{H}, \mathrm{m}), 2.11-2.19(2 \mathrm{H}, \mathrm{m}), 2.37(1 \mathrm{H}, \mathrm{td}, J=11.7,3.6 \mathrm{~Hz}), 2.42(1 \mathrm{H}, \mathrm{d}$, $J=17.2 \mathrm{~Hz}), 2.49-2.55(2 \mathrm{H}, \mathrm{m}), 2.72(1 \mathrm{H}, \mathrm{ddd}, J=10.9,5.7,1.7 \mathrm{~Hz}), 2.79-2.85(1 \mathrm{H}, \mathrm{m}), 3.09(1 \mathrm{H}, \mathrm{ddd}, J=15.6,11.0,5.0 \mathrm{~Hz}), 3.16$

(1H, d, $J=11.5 \mathrm{~Hz}), 3.34(3 \mathrm{H}, \mathrm{s}), 3.43(3 \mathrm{H}, \mathrm{s}), 3.45(3 \mathrm{H}, \mathrm{s}), 4.95-5.04(2 \mathrm{H}, \mathrm{m}), 5.47(1 \mathrm{H}, \mathrm{ddd}, J=17.8,10.0,8.9 \mathrm{~Hz}), 6.44(1 \mathrm{H}, \mathrm{s})$, $6.76(1 \mathrm{H}, \mathrm{s})$.

${ }^{13} \mathrm{C}-\mathrm{NMR}\left(\mathrm{C}_{6} \mathrm{D}_{6}\right) \delta ; 30.3,38.2,38.4,39.1,47.5,51.3,52.7,56.0,56.4,62.5,63.1,110.5,113.1,117.3,127.7,130.9,140.2,148.9$, $149.2,173.3$.

Anal. Calcd for $\mathrm{C}_{20} \mathrm{H}_{27} \mathrm{NO}_{4}$ : C,69.54; H, 7.88; N, 4.05. Found: C, 69.45, H, 8.03; N, 3.84 .

HR-FAB MS : Calcd for $\mathrm{C}_{20} \mathrm{H}_{28} \mathrm{NO}_{4}[\mathrm{M}+\mathrm{H}]^{+}$: 346.2018. Found 346.2031.

The synthesis of (3-ethyl-9,10-dimethoxy-1,3,4,6,7,11b-hexahydro-2H-pyrido[2,1-a]isoquinolin-2-yl)acetic acid methyl ester (10). To the methanol solution (5 ml) of compound 9 was added Pd (14.5 mg, $0.014 \mathrm{mmol})$, and the mixture was allowed to react for $16 \mathrm{~h}$ under $\mathrm{H}_{2}$ at room temperature. Then the solution was filtrated using celite, and the filtrate was evaporated off to leave a residue, which was chromatographed on silica gel $(\mathrm{AcOEt} / \mathrm{MeOH}=10)$ to give $10(40.9 \mathrm{mg}, 87 \%)$. The optical purity was determined by HPLC (CHIRALCEL OD, hexane $/ \mathrm{i}-\mathrm{PrOH}=3,0.5 \mathrm{ml} / \mathrm{min}$, retention times $13.8 \mathrm{~min}$ and $18.8 \mathrm{~min}$ ). 


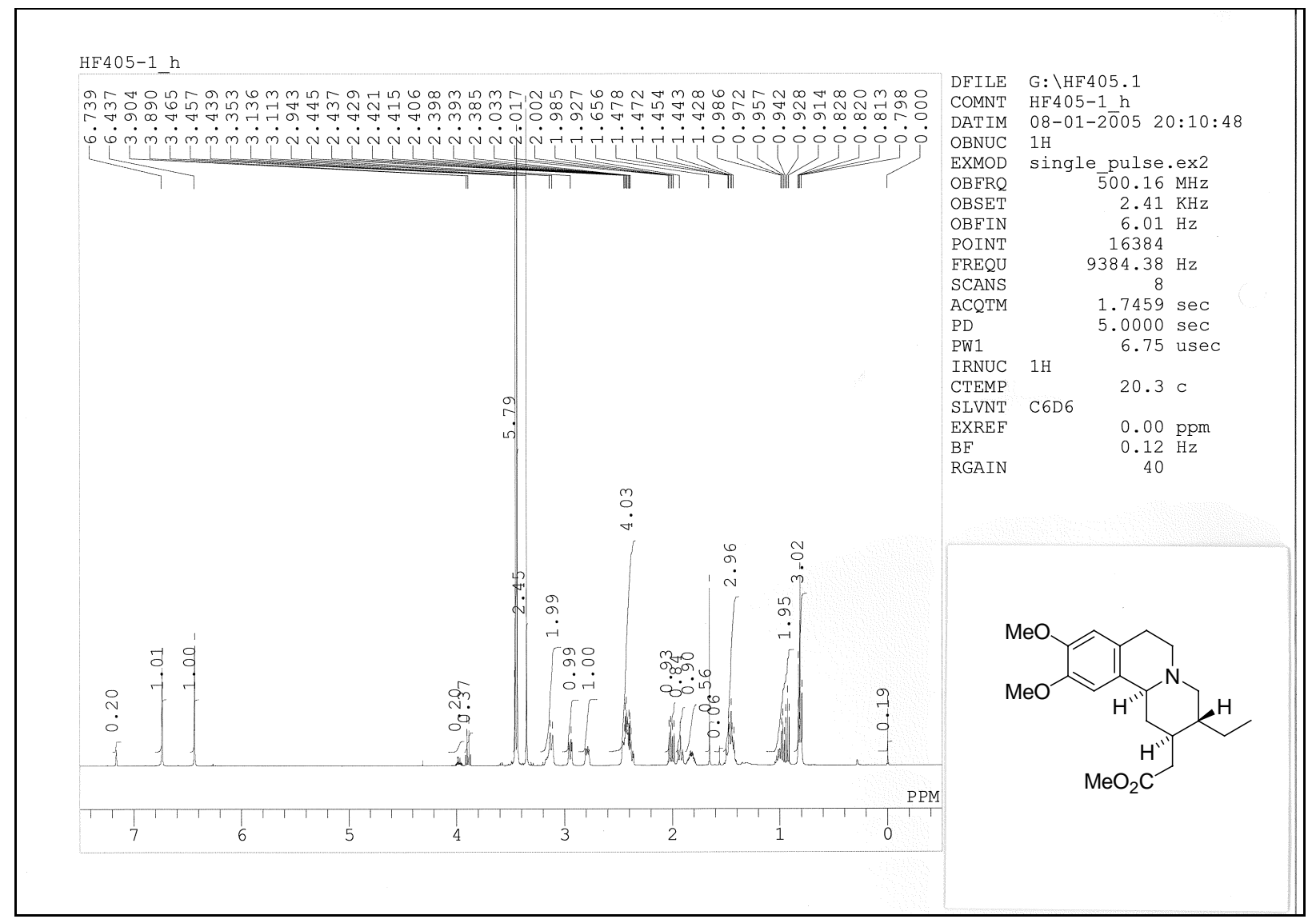

(3-ethyl-9.10-dimethoxy-1,3,4,6,7,11b-hexahydro-2H-pyrido[2,1-a]isoquinolin-2-yl)acetic acid methyl ester

Yield $95 \%$ yellow crystal : $\mathrm{mp} 65-75^{\circ} \mathrm{C}$

$R-(98.5 \%$ ee $):[\alpha]^{18}{ }_{\mathrm{D}}+85.5\left(\mathrm{c} 0.76, \mathrm{CHCl}_{3}\right)$

$S-(99.7 \%$ ee $):[\alpha]^{17} \mathrm{D}-87.2\left(c 1.66, \mathrm{CHCl}_{3}\right)$

${ }^{1} \mathrm{H}-\mathrm{NMR}\left(\mathrm{C}_{6} \mathrm{D}_{6}\right) \delta: 0.82(3 \mathrm{H}, \mathrm{t}, J=7.3 \mathrm{~Hz}), 1.00-1.04(1 \mathrm{H}, \mathrm{m}), 1,39-1.51(3 \mathrm{H}, \mathrm{m}), 1.79-1.88(1 \mathrm{H}, \mathrm{m}), 1,93(1 \mathrm{H}, \mathrm{t}, J=11.0 \mathrm{~Hz}), 2.02$

(1H, dd, t=15.5, 8.4 Hz), 2.37-2.46 (4H, m), 2.75-2.80 (1H, m), $2.93(1 \mathrm{H}, \mathrm{dd}, J=11.3,3.8 \mathrm{~Hz}), 3.10-3.17(2 \mathrm{H}, \mathrm{m}), 3.36(3 \mathrm{H}, \mathrm{s})$,

$3.45(3 \mathrm{H}, \mathrm{s}), 3.47(3 \mathrm{H}, \mathrm{s}), 6.46(1 \mathrm{H}, \mathrm{s}), 6.76(1 \mathrm{H}, \mathrm{s})$.

${ }^{13} \mathrm{C}-\mathrm{NMR}\left(\mathrm{C}_{6} \mathrm{D}_{6}\right) \delta ; 11.2,23.7,30.0,38.5,38.7,41.8,50.9,52.8,55.6,56.0,61.4,63.0,110.1,112.8,127.4,130.7,148.5,148.7$, 173.0.

HR-FAB MS : Calcd for $\mathrm{C}_{20} \mathrm{H}_{30} \mathrm{NO}_{4}[\mathrm{M}+\mathrm{H}]^{+}$: 348.2175. Found 348.2191. 
The synthesis of $\quad N$-[2-(3,4-dimethoxyphenyl)ethyl]-2-(3-ethyl-9,10-dimethoxy-1,3,4,6,7,11b-hexa-hydro-2Hpyrido[2,1-a]- isoquinolin-2-yl)acetamide. To the benzene solution $(1 \mathrm{ml})$ of 2-(3,4-dimethoxyphenyl)ethylamine ( $85 \mu \mathrm{l}, 0.5$ mmol) was added $\mathrm{AlMe}_{3}(500 \mu \mathrm{l}, 1 \mathrm{mmol})$ dropwise, and the mixture was allowed to stir for $1 \mathrm{~h}$ at room temperature. Then the benzene solution $(3 \mathrm{ml})$ of compound $\mathbf{1 0}$ was added, and the mixture was reacted for $10 \mathrm{~h}$ at $115^{\circ} \mathrm{C}$. After cooling, $\mathrm{H}_{2} \mathrm{O}(10 \mathrm{ml})$ was added, and the mixture was filtrated using celite to give the filtrate, which was extracted with AcOEt. The celite was washed with $10 \% \mathrm{HCl}(8 \mathrm{ml})$, which was basified with sat. $\mathrm{NaHCO}_{3}$, and the aqueous layer thus obtained was extracted with AcOEt. The organic layer was combined, dried over MgSO4, and evaporated to leave a residue, which was chromatographed on silica gel $\left(\mathrm{CH}_{2} \mathrm{Cl}_{2} / \mathrm{MeOH}=20\right.$ to 8$)$ to give the product (35.3 mg, $\left.73 \%\right)$.

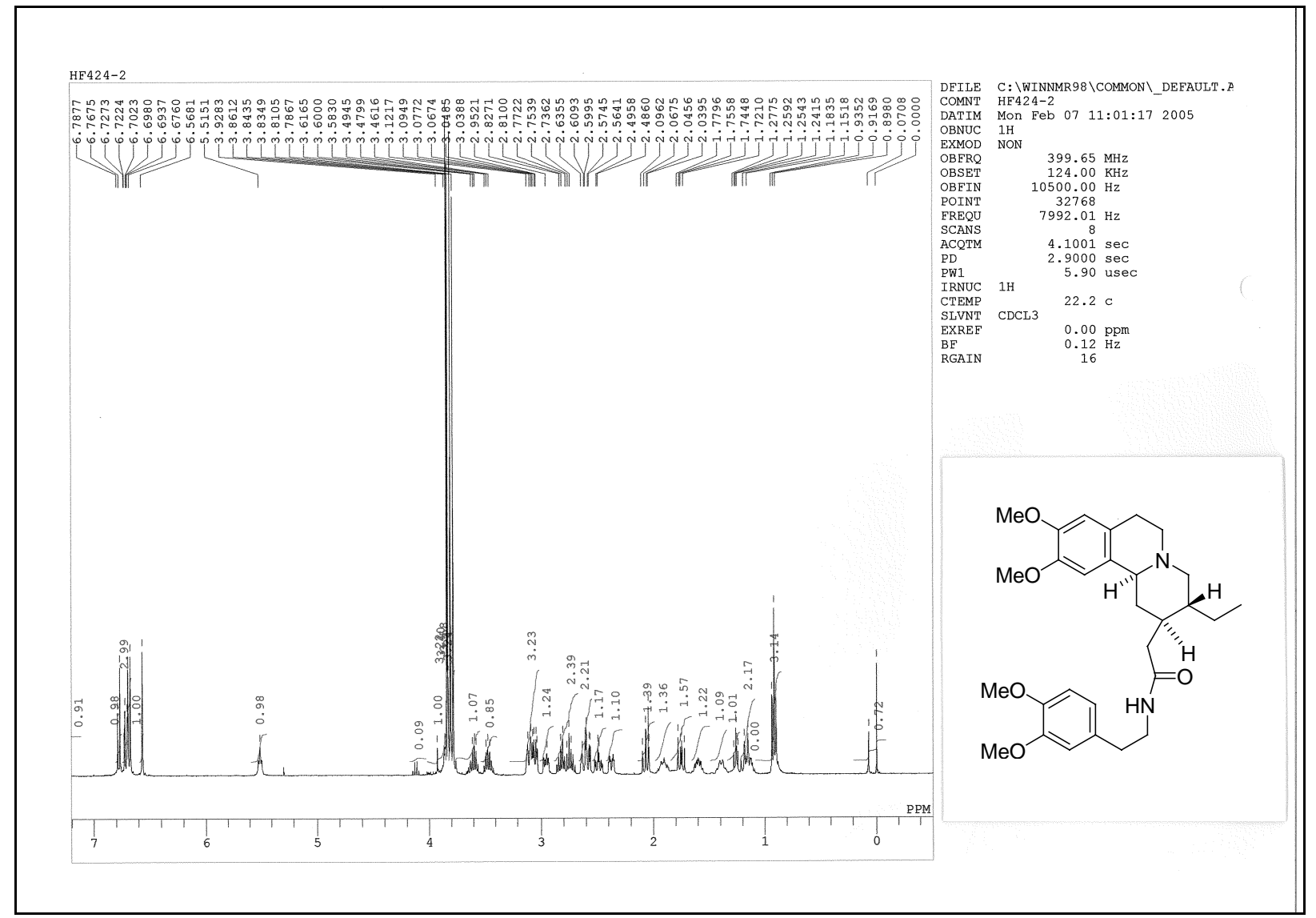

$N$-[2-(3,4-dimethyoxyphenyl)ethyl]-2-(3-ethyl-9,10-dimethoxy-1,3,4,6,7,11b-hexahydro-2H-pyrido[2,1-a]isoquinoline-2-yl )acetamide

Yield $73 \%$ colarless crystal : mp $165-168{ }^{\circ} \mathrm{C}$

$R-(98.5 \%$ ee $):[\alpha]^{18}{ }^{18}+85.5\left(c 0.76, \mathrm{CHCl}_{3}\right)$

$S$ - $(99.7 \%$ ee $):[\alpha]^{17}{ }^{D}-87.2\left(c 1.66, \mathrm{CHCl}_{3}\right)$ 
${ }^{1} \mathrm{H}-\mathrm{NMR}\left(\mathrm{CDCl}_{3}\right) \delta: 0.92(3 \mathrm{H}, \mathrm{t}, J=7.5 \mathrm{~Hz}), 1.11-1.23(2 \mathrm{H}, \mathrm{m}), 1.39-1.42(1 \mathrm{H}, \mathrm{m}), 1.61(1 \mathrm{H}, \mathrm{ddd}, J=13.7,7.6,2.9 \mathrm{~Hz}), 1.75(1 \mathrm{H}$, dd, $J=14.1,9.5 \mathrm{~Hz}), 1.88-1.94(1 \mathrm{H}, \mathrm{m}), 2.08(1 \mathrm{H}, \mathrm{d}, J=11.2 \mathrm{~Hz}), 2.38(1 \mathrm{H}, \mathrm{dt}, J=12.6,3.1 \mathrm{~Hz}), 2.50(1 \mathrm{H}, \mathrm{td}, J=11.4,4.0 \mathrm{~Hz}), 2.60$ (1H, dd, $J=14.0,4.0 \mathrm{~Hz}), 2.64$ (1H, bs), $2.74(1 \mathrm{H}$, qui, $J=6.9 \mathrm{~Hz}), 2.83$ (1H, qui, $J=6.9 \mathrm{~Hz}), 2.97(1 \mathrm{H}, \mathrm{dd}, J=10.5,5.4 \mathrm{~Hz}), 3.07$ $(1 \mathrm{H}, \mathrm{dd}, J=11.6,4.0 \mathrm{~Hz}), 3.11(2 \mathrm{H}, \mathrm{d}, J=10.7 \mathrm{~Hz}), 3.43-3.51(1 \mathrm{H}, \mathrm{m}), 3.57-3.65(1 \mathrm{H}, \mathrm{m}), 3.79(3 \mathrm{H}, \mathrm{s}), 3.81(3 \mathrm{H}, \mathrm{s}), 3.83(3 \mathrm{H}, \mathrm{s})$, $3.84(3 \mathrm{H}, \mathrm{s}), 5.53(1 \mathrm{H}, \mathrm{t}, J=5.9 \mathrm{~Hz}), 6.57(1 \mathrm{H}, \mathrm{s}), 6.69-6.73(2 \mathrm{H}, \mathrm{m}), 6.78(1 \mathrm{H}, \mathrm{d}, J=8.0 \mathrm{~Hz})$.

${ }^{13} \mathrm{C}-\mathrm{NMR}\left(\mathrm{CDCl}_{3}\right) \delta ; 12.0,24.4,30.0,36.5,38.0,39.0,41.7,42.2,42.6,53.2,56.69,56.70,56.8,56.9,61.9,63.3,109.1,112.2$, $112.3,112.7,121.4,127.5,130.6,132.0,148.1,148.4,148.6,149.9,173.2$.

HR-FAB MS : Calcd for $\mathrm{C}_{29} \mathrm{H}_{41} \mathrm{~N}_{2} \mathrm{O}_{5}[\mathrm{M}+\mathrm{H}]^{+}$: 497.3015. Found 497.3009.

Synthesis of O-methylpsychotrine. To the benzene solution (4.0 ml) of $N$-[2-(3,4-dimethyoxyphenyl)-ethyl]-2-(3-ethyl-9,10dimethoxy-1,3,4,6,7,11b-hexahydro-2H-pyrido[2,1-a]isoquinoline-2-yl)acetamide (30.2 mg, $0.061 \mathrm{mmol}$ ) was added $\mathrm{POCl}_{3}$ (50 $\mu 1,0.79 \mathrm{mmol})$, and the mixture was allowed to react for $3.8 \mathrm{~h}$ at $110{ }^{\circ} \mathrm{C}$. Then $\mathrm{AcOEt}(10 \mathrm{ml})$ and $1 \mathrm{M} \mathrm{NaOH}(7 \mathrm{ml})$ were added to the mixture, and the organic layer was separated. The aqueous layer was extracted with AcOEt, and the combined organic layer was dried over $\mathrm{MgSO} 4$, and evaporated off. The residue thus obtained was chromatographed on silica gel $\left(\mathrm{CH}_{2} \mathrm{Cl}_{2} / \mathrm{MeOH}=10\right.$ to 1$)$ to give 2-(6,7-Dimethoxy-3,4-dihydro-isoquinolin-1-ylmethyl)-3-ethyl-9,10-dimethoxy-1,3,4,6,7,11bhexahydro-2H-pyrido[2,1-a]isoquinoline (O-methylpsychotorine) (21.9 mg, 75\%). 


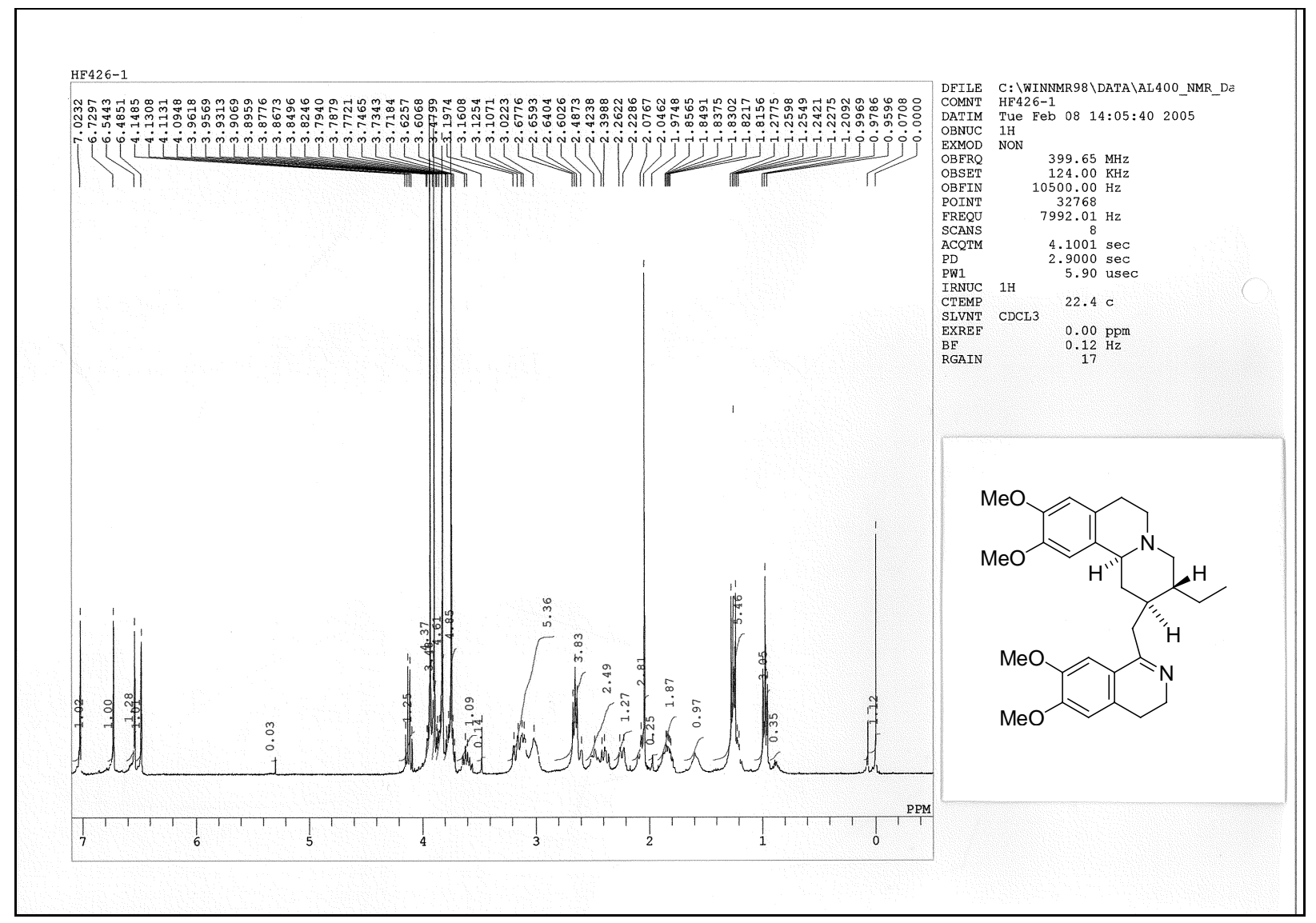

$O$-methylpsychotorine

Yield $75 \%$ yellow oil

$R-(98.5 \%$ ee $):[\alpha]^{18}+85.5\left(c 0.76, \mathrm{CHCl}_{3}\right)$

$S-(99.7 \%$ ee $):[\alpha]^{17}$ D $-87.2\left(c 1.66, \mathrm{CHCl}_{3}\right)$

${ }^{1} \mathrm{H}-\mathrm{NMR}\left(\mathrm{CDCl}_{3}\right) \delta: 0.98(3 \mathrm{H}, \mathrm{t}, J=7.5 \mathrm{~Hz}), 1.19-1.28(2 \mathrm{H}, \mathrm{m}), 1.60(1 \mathrm{H}, \mathrm{bs}), 1.80-1.92(2 \mathrm{H}, \mathrm{m}), 2.04(1 \mathrm{H}, \mathrm{t}, J=11.1 \mathrm{~Hz}), 2.25(1 \mathrm{H}$, d, $J=13.4 \mathrm{~Hz}), 2.36(1 \mathrm{H}, \mathrm{dd}, J=14.2,10.0 \mathrm{~Hz}), 2.46-2.51(1 \mathrm{H}, \mathrm{m}), 2.60(1 \mathrm{H}, \mathrm{bs}), 2.66(2 \mathrm{H}, \mathrm{t}, J=7.4 \mathrm{~Hz}), 2.98-3.20(5 \mathrm{H}, \mathrm{m})$, 3.57-3.65 (1H, m), 3.72-3.79 (1H, m), $3.75(3 \mathrm{H}, \mathrm{s}), 3.82(3 \mathrm{H}, \mathrm{s}), 3.93(3 \mathrm{H}, \mathrm{s}), 6.49(1 \mathrm{H}, \mathrm{s}), 6.54(1 \mathrm{H}, \mathrm{s}), 6.73(1 \mathrm{H}, \mathrm{s}), 7.02(1 \mathrm{H}, \mathrm{s})$.

${ }^{13} \mathrm{C}-\mathrm{NMR}\left(\mathrm{CDCl}_{3}\right) \delta ; 11.3,23.8,26.1,29.0,38.3,39.3,39.7,42.4,46.9,52.5,55.7,55.8,56.0,56.2,61.2,62.4,108.1,108.9,110.3$, $111.3,122.3,126.5,129.9,131.5,146.9,147.3,147.5,150.8,166.3$.

HR-FAB MS : Calcd for $\mathrm{C}_{29} \mathrm{H}_{39} \mathrm{~N}_{2} \mathrm{O}_{4}[\mathrm{M}+\mathrm{H}]^{+}$: 479.2910. Found 479.2899. 
The synthesis of emetine. To the DMF solution $(0.4 \mathrm{ml})$ of O-methylpsychotrine $(11.0 \mathrm{mg}, 0.023$ mmol $)$ were added chloro[(1S,2S)- $N$-(p-toluenesulfonyl)-1,2-diphenylethanediamine](p-cymene)ruthenium(II) $(1.0 \mathrm{mg}, 5 \mathrm{~mol} \%), \mathrm{HCO}_{2} \mathrm{H}(50 \mu \mathrm{l}$, $1.33 \mathrm{mmol})$, and $\mathrm{Et}_{3} \mathrm{~N}(20 \mu \mathrm{l}, 0.15 \mathrm{mmol})$, and the mixture was allowed to react for $3.3 \mathrm{~h}$ at room temperature. The mixture was diluted with AcOEt $(10 \mathrm{ml})$, and washed with sat. $\mathrm{NaHCO}_{3}(5 \mathrm{ml})$ and $\mathrm{H}_{2} \mathrm{O}(3 \mathrm{ml})$. The aqueous layer was extracted with AcOEt and $\mathrm{CH}_{2} \mathrm{Cl}_{2}$, then the combined organic layer was dried over MgSO4, and evaporated. The residue thus obtained was chromatographed on $\mathrm{NH}$ silica gel $\left(\mathrm{CH}_{2} \mathrm{Cl}_{2} / \mathrm{MeOH}=20\right)$ to give the product $(7.9 \mathrm{mg}, 72 \%)$.

$R-11(98.6 \%$ ee $):[\alpha]_{\mathrm{D}}+45.8\left(c 0.66, \mathrm{CHCl}_{3}\right)$

$S-11(99.7 \%$ ee $):[\alpha]_{\mathrm{D}}-46.2\left(c 0.13, \mathrm{CHCl}_{3}\right)$

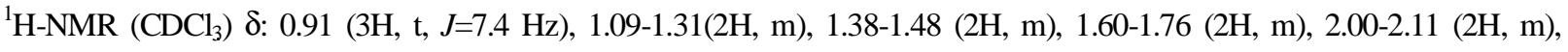
2.50-2.54 (1H, m), 2.54-2.66 (2H, m), $2.73(2 \mathrm{H}, \mathrm{t}, J=5.6 \mathrm{~Hz}), 3.00-3.12(4 \mathrm{H}, \mathrm{m}), 3.16(1 \mathrm{H}, \mathrm{br} \mathrm{d}, J=9.6 \mathrm{~Hz}), 3.23(1 \mathrm{H}, \mathrm{dt}, J=13.2$, $6.4 \mathrm{~Hz}), 3.81(3 \mathrm{H}, \mathrm{s}), 3.847(6 \mathrm{H}, \mathrm{s}), 3.85(3 \mathrm{H}, \mathrm{s}), 4.11(1 \mathrm{H}, \mathrm{d} J=12.0 \mathrm{~Hz}), 6.53(1 \mathrm{H}, \mathrm{s}), 6.58(1 \mathrm{H}, \mathrm{s}), 6.59(1 \mathrm{H}, \mathrm{s}), 6.75(1 \mathrm{H}, \mathrm{s})$.

${ }^{13} \mathrm{C}-\mathrm{NMR}\left(\mathrm{CDCl}_{3}\right) \delta: 11.2,23.6,29.3,29.4,36.8,37.0,40.1,40.8,41.7,51.8,52.3,55.8,55.9,56.0,56.2,61.4,62.4,108.6,109.2$, $111.5,111.8,126.9,127.0,130.2,131.6,147.2,147.3,147.3,147.5$.

HR-FAB MS:Calcd for $\mathrm{C}_{29} \mathrm{H}_{41} \mathrm{~N}_{2} \mathrm{O}_{4}[\mathrm{M}+\mathrm{H}]^{\square}:$ 481.3066. Found 481.3070. 